Modern Physics Letters A

(C) World Scientific Publishing Company

\title{
The effects of the dark energy on the static Schrödinger-Newton system - an Adomian Decomposition Method and Padé approximants based approach
}

\author{
Man Kwong Mak \\ Departamento de Física, Facultad de Ciencias Naturales, Universidad de Atacama, Copayapu \\ 485, Copiapó, Chile, \\ email: mankwongmak@gmail.com \\ Chun Sing Leung \\ Department of Applied Mathematics, Hong Kong Polytechnic University, Hong Kong, Hong \\ Kong SAR, P. R. China, \\ email: chun-sing-hkpu.leung@polyu.edu.hk \\ Tiberiu Harko \\ Astronomical Observatory, 19 Ciresilor Street, 400487 Cluj-Napoca, Romania, \\ email: tiberiu.harko@aira.astro.ro \\ Department of Physics, Babes-Bolyai University, Kogalniceanu Street, Cluj-Napoca 400084, \\ Romania, \\ School of Physics, Sun Yat-Sen University, Guangzhou 510275, People's Republic of China
}

Received (25/092020)

Revised $(01 / 12 / 2020)$

\begin{abstract}
The Schrödinger-Newton system is a nonlinear system obtained by coupling together the linear Schrödinger equation of quantum mechanics with the Poisson equation of Newtonian mechanics. In the present work we will investigate the effects of a cosmological constant (dark energy or vacuum fluctuation) on the Schrödinger-Newton system, by modifying the Poisson equation through the addition of a new term. The corresponding Schrödinger-Newton- $\Lambda$ system cannot be solved exactly, and therefore for its study one must resort to either numerical or semianalytical methods. In order to obtain a semianalytical solution of the system we apply the Adomian Decomposition Method, a very powerful method used for solving a large class of nonlinear ordinary and partial differential equations. Moreover, the Adomian series are transformed into rational functions by using the Padé approximants. The semianalytical approximation is compared with the full numerical solution, and the effects of the dark energy on the structure of the Newtonian quantum system are investigated in detail.
\end{abstract}

Keywords: Schrödinger-Newton system; dark energy; Adomian Decomposition Method; series solutions

PACS Nos.: 04.50.Kd, 04.20.Cv, 04.20.Fy 


\section{Introduction}

The search for quantum gravity is one of the major directions of research in theoretical physics. There are many proposals for building a quantum theory of gravity, but achieving this goal seems to be still far away. For recent reviews of the present status of quantum gravity see 1 - 1 However, the difficulties of quantizing general relativity, and of quantum field theory in curved geometries have led to the suggestion that perhaps a satisfactory description of quantum gravity may be obtained from the unification of quantum mechanics and Newtonian gravity $\stackrel{5}{5}$ Hence, in this approach, the basic equations of quantum gravity can be formulated as

$$
i \hbar \frac{\partial \psi(\vec{r}, t)}{\partial t}=-\frac{\hbar^{2}}{2 m} \Delta \psi(\vec{r}, t)+m \Phi(\vec{r}, t) \psi(\vec{r}, t),
$$

and

$$
\Delta \Phi(\vec{r}, t)=4 \pi G \rho_{m}(\vec{r}, t),
$$

respectively, where $\hbar$ is Planck's constant, $G$ is the gravitational constant, $m$ is the particle mass, $\psi(\vec{r}, t)$ is the particle wave function, $\Phi(\vec{r}, t)$ is the gravitational potential, satisfying the Poisson equation (2), and $\rho_{m}(\vec{r}, t)$ is the mass density. As for the gravitational potential one must assume, in this formulation, that it is a stochastic quantity, with moments given by $\langle\Phi(\vec{r}, t)\rangle=\Phi_{c l}(\vec{r}, t)$, and $\Phi(\vec{r}, t) \Phi\left(\vec{r}^{\prime}, t^{\prime}\right)-\Phi\left(\vec{r}^{\prime}, t^{\prime}\right) \Phi(\vec{r}, t)=\left(\hbar G /\left|\vec{r}-\vec{r}^{\prime}\right|\right) \delta\left(t-t^{\prime}\right) \stackrel{5}{\text { g }}$ respectively. The averaged value of the gravitational potential can be obtained as $\langle\Phi\rangle \sim \sqrt{\hbar^{2} G / m R^{3}}$, which gives, by taking into account that in the Newtonian limit $\langle\Phi\rangle=G m / R$, the condition $m^{3} R \sim \hbar^{2} / G$. Hence it turns out that the particle behavior is genuinely quantum if the condition $m^{3} R<<\hbar^{2} / G \approx 10^{-47} \mathrm{~cm} \mathrm{~g}^{3}$ is satisfied.

By assuming that the mass density can be represented as $\rho_{m}(\vec{r}, t)=m|\psi(\vec{r}, t)|^{2}$, the system of equations (11) and (2) becomes the so-called Schrödinger-Newton (or Schrödinger-Poisson) system, whose properties have been intensively investigated $[-17$ The Schrödinger-Newton system can also be obtained immediately as the nonrelativistic limit of the semi-classical theory of gravity, that is, the theory in which the gravitational field is considered classical, while the matter part is quantized, with the field equations given by 18

$$
R_{\mu \nu}-\frac{1}{2} g_{\mu \nu} R=\frac{8 \pi G}{c^{4}}\left\langle\psi\left|\hat{T}_{\mu \nu}\right| \psi\right\rangle,
$$

where $\hat{T}_{\mu \nu}$ is the quantum mechanical operator associated to the energy-momentum tensor, with its expectation value computed by choosing some appropriate quantum state. The Schrödinger-Newton system can be reduced to a single differentialintegral equation, given by $[9$

$$
i \hbar \frac{\partial \psi(\vec{r}, t)}{\partial t}=-\frac{\hbar^{2}}{2 m} \nabla^{2} \psi(\vec{r}, t)-G m^{2} \int \frac{\left|\psi\left(\vec{r}^{\prime}, t\right)\right|^{2}}{\left|\vec{r}-\vec{r}^{\prime}\right|} \psi(\vec{r}, t) d \vec{r}^{\prime} .
$$

The Schrödinger-Newton system with both local and nonlocal nonlinearities was investigated numerically in, $\frac{19}{19}$ by also including in the model the modifications of the 
gravitational force due to the non-minimal coupling between curvature and matter, and by using the numerical solvers developed for studying light propagating in the $\mathrm{S}-\mathrm{N}$ model.

Recently, an extension of the standard Schrödinger-Newton system was proposed and investigated in $\stackrel{20}{, 2 y}$ including in the mathematical formalism the effects of the dark energy, represented by a cosmological constant $\Lambda$. Presently, it is assumed that dark energy drives the late-time acceleration of the Universe, and plays a determin-

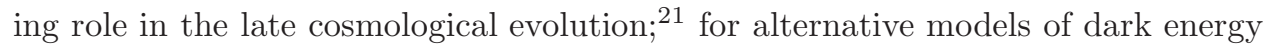
as modified gravity see $\frac{22}{22}$ and references therein. In 20 the regime in which dark energy dominates both canonical quantum diffusion as well as gravitational selfattraction was investigated in detail by numerically solving Eq. (4). It was found that the dark energy domination regime occurs for sufficiently delocalized objects with an arbitrary mass. Moreover, one must also note that a minimal delocalization width of about $67 \mathrm{~m}$ was determined from the high precision numerical analysis. The modifications of an initially spherical Gaussian wave packet induced by the presence of a positive cosmological constant and of the gravitational field were also investigated. It turns out that the order of magnitude of the radial distance separating the collapsing phase from the expansionary one is consistent with the analytical estimates obtained for the classical turnaround radius for a spherically symmetric massive object in the presence of dark energy. However, the physical time required to detect experimentally these modifications is very large, and therefore they can be measured only in physical systems containing a high effective cosmological constant (dark energy), or, alternatively, via their effects in a stationary Universe.

It is the goal of the present Letter to investigate the mathematical and physical properties of the static Schrödinger-Newton system in the presence of dark energy, modeled as a cosmological constant. We call the corresponding mathematical and physical model as the Schrödinger-Newton- $\Lambda(\mathrm{S}-\mathrm{N}-\Lambda)$ system, and it represents a natural generalization of the standard Schrödinger-Newton model of quantum gravity. In order to gain a better understanding of the physical and mathematical properties of the $\mathrm{S}-\mathrm{N}-\Lambda$ system we will also obtain some semianalytical solutions of it, by using the Adomian Decomposition Method. The Adomian Decomposition Method is a powerful mathematical technique introduced in, 23, 26 and which was applied for obtaining solutions of a large class of nonlinear ordinary, stochastic, and partial differential equations, or of integral equations, $27 \sqrt[38]{38}$ with applications in various scientific fields.

In our present approach we first reformulate the static Schrödinger-Newton- $\Lambda$ equations as a system of two integral equations, and we solve them by expanding the nonlinear terms by using the Adomian polynomials.23 26 This allows us to obtain a series solution of the $\mathrm{S}-\mathrm{N}-\Lambda$ system. In order to avoid the possible oscillatory or singular behavior of the solution we will represent the Adomian series with the help of their Padé approximants. The semianalytical results are compared with the full numerical solutions for a large range of values of the effective cosmological constant. We find that the Adomian-Padé type solutions give a good description of 
M. K. Mak, C. S. Leung, T. Harko

the numerical results for the static Schrödinger-Newton system, and thus they can offer some new insights into the important problem of the quantization of gravity for static spherically systems at the Newtonian level.

The present Letter is organized as follows. In Section 2 we present the Schrödinger-Newton- $\Lambda$ system, and obtain its integral representation. The Adomian Decomposition Method, as well as the Padé approximation is also briefly introduced. We obtain the semianalytical solution of the Schrödinger-Newton- $\Lambda$ system for arbitrary initial conditions in Section 3 , The comparison of the Adomian Decomposition Method results with the full numerical solution is performed in Section 4 . We discuss and conclude our results in Section 5 .

\section{The static Schrödinger-Newton- $\Lambda$ system, and its integral representation}

In the present Section we introduce the Schrödinger-Newton- $\Lambda$ system, and we present its dimensionless form. Moreover, we will obtain the integral equations representation of the system. We also briefly introduce the Adomian Decomposition Method for systems of ordinary nonlinear differential equations.

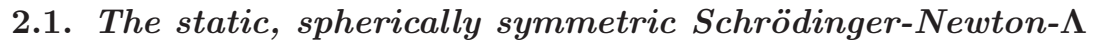 system}

For a single particle system the static Schrödinger-Newton- $\Lambda$ system takes the form 20

$$
\begin{gathered}
-\frac{\hbar^{2}}{2 m} \Delta \psi(\vec{r})+m \Phi(\vec{r}) \psi(\vec{r})=E \psi(\vec{r}), \\
\Delta \Phi(\vec{r})=4 \pi G m|\psi(\vec{r})|^{2}-\Lambda c^{2},
\end{gathered}
$$

where $E$ is the energy eigenvalue of the particle, while the constant term $\Lambda$ models the effects of the dark energy, or, alternatively, of the vacuum fluctuations. In the case $\Lambda \equiv 0$, the system of equations (5) and (6) reduces to the standard static Schrödinger-Newton system, whose interesting properties have been investigated in detail $\sqrt[39]{46}$ In the following we will assume, without any loss of generality, that the wave function $\psi$ is real. In order to obtain a simpler form of the S-N- $\Lambda$ system, we introduce two new functions $S$ and $V$, defined as $\psi(\vec{r})=\left(\frac{\hbar^{2}}{8 \pi G m^{3}}\right)^{1 / 2} S(\vec{r})$, and $E-m \Phi=\frac{\hbar^{2}}{2 m} V(\vec{r})$, respectively $\underline{40} \underline{41}$. Both $S$ and $V$ have the physical units of $1 /$ length $^{2}$. Then the Schrödinger-Newton- $\Lambda$ system takes the form

$$
\begin{gathered}
\Delta S=-S V, \\
\Delta V=-S^{2}+\lambda,
\end{gathered}
$$

where we have denoted

$$
\lambda=\frac{2 m^{2} c^{2}}{\hbar^{2}} \Lambda=4.522 \times 10^{-29} \times\left(\frac{m}{m_{p}}\right)^{2} \times\left(\frac{\Lambda}{10^{-56} \mathrm{~cm}^{-2}}\right) \mathrm{cm}^{-4}
$$


where $m_{p}$ denotes the mass of the proton.

Eq. (5) can be derived from the variational principle, $\underline{43}$

$$
H[\psi(\vec{r})]=\frac{\hbar^{2}}{2 m} \int\left[|\nabla \psi(\vec{r})|^{2}+\frac{m}{4} \Phi(\vec{r})|\psi(\vec{r})|^{2}\right] d^{3} \vec{r},
$$

with the wave function satisfying the normalization condition $\int|\psi(\vec{r})|^{2} d^{3} \vec{r}=1$. The Poisson equation (6) can be solved by using a Green function techniques to $\operatorname{give} e^{20}$

$$
\Phi(\vec{r})=-G m \int \frac{|\psi(\vec{r})|^{2}}{\left|\vec{r}-\vec{r}^{\prime}\right|} d^{3} \vec{r}^{\prime}+\frac{\Lambda c^{2}}{4 \pi} \int \frac{1}{\left|\vec{r}-\vec{r}^{\prime}\right|} d^{3} \vec{r}^{\prime} .
$$

Hence, the Schrödinger-Newton- $\Lambda$ system can be obtained as an extremum of the functional

$$
\begin{aligned}
H[\psi(\vec{r})]= & \frac{\hbar^{2}}{2 m} \int|\nabla \psi(\vec{r})|^{2} d^{3} \vec{r}-G m^{2} \iint \frac{|\psi(\vec{r})|^{2}\left|\psi\left(\vec{r}^{\prime}\right)\right|^{2}}{\left|\vec{r}-\vec{r}^{\prime}\right|} d^{3} \vec{r} d^{3} \vec{r}^{\prime}+ \\
& \frac{m c^{2}}{4 \pi} \Lambda \iint \frac{|\psi(\vec{r})|^{2}}{\left|\vec{r}-\vec{r}^{\prime}\right|} d^{3} \vec{r} d^{3} \vec{r}^{\prime} .
\end{aligned}
$$

In the following we will limit our investigations to the spherically symmetric case, with $S=S(r)$ and $V=V(r)$, respectively, where $r$ is the radius vector. Moreover, for the wave function normalization as well as for the energy eigenvalues we will adopt the same prescription as in the case of the simple Schrödinger-Newton system, namely, $\int_{0}^{\infty} r^{2} S^{2} d r=\frac{2 G m^{3}}{\hbar^{2}}$, and $E=\frac{\hbar^{2}}{2 m} \lim _{r \rightarrow \infty} V(r)$, respectively 40 [41

In the case of spherical symmetry the Schrödinger-Newton- $\Lambda$ system can be written as

$$
\begin{gathered}
\frac{1}{r} \frac{d^{2}}{d r^{2}}[r S(r)]=S^{\prime \prime}(r)+\frac{2}{r} S^{\prime}(r)=-S(r) V(r), \\
\frac{1}{r} \frac{d^{2}}{d r^{2}}[r V(r)]=V^{\prime \prime}(r)+\frac{2}{r} V^{\prime}(r)=-S^{2}(r)+\lambda .
\end{gathered}
$$

The system of equations (13) and (14) is invariant with respect to the transformations $S \rightarrow S / a^{2}, V \rightarrow V / a^{2}, r \rightarrow a r$, and $\lambda \rightarrow \lambda / a^{4}$, respectively, where $a=$ constant. In the following we will consider the system (13)-(14) with the initial conditions $S(0) \neq 0$ and $V(0) \neq 0$, implying that the functions $S(r)$ and $V(r)$ are smooth and finite at the origin $r=0$, and $S^{\prime}(0)=0$ and $V^{\prime}(0)=0$, respectively, that is, with vanishing derivatives of $S$ and $V$ at the origin.

\subsection{Integral equation formulation of the Schrödinger-Newton- $\Lambda$ system}

By integrating once Eqs. (13) and (14) we obtain

$$
\frac{d}{d r}[r S(r)]=S_{0}-\int_{0}^{r} x^{\prime} S\left(x^{\prime}\right) V\left(x^{\prime}\right) d x^{\prime}
$$


and

$$
\frac{d}{d r}[r V(r)]=V_{0}+\frac{\lambda r^{2}}{2}-\int_{0}^{r} x^{\prime} S^{2}\left(x^{\prime}\right) d x^{\prime},
$$

respectively. A second integration gives

$$
\begin{aligned}
& r S(r)=S_{0} r-\int_{0}^{r} \int_{0}^{x^{\prime \prime}} x^{\prime} S\left(x^{\prime}\right) V\left(x^{\prime}\right) d x^{\prime} d x^{\prime \prime}, \\
& r V(r)=V_{0} r+\frac{\lambda r^{3}}{6}-\int_{0}^{r} \int_{0}^{x^{\prime \prime}} x^{\prime} S^{2}\left(x^{\prime}\right) d x^{\prime} d x^{\prime \prime} .
\end{aligned}
$$

By using the Cauchy formula for repeated integration, $\int_{a}^{x} \int_{a}^{x_{1}} \ldots \int_{a}^{x_{n-1}} f\left(x_{n}\right) d x_{n} \ldots d x_{2} d x_{1}=[1 /(n-1) !] \int_{a}^{x}(x-t)^{n-1} f(t) d t$, we finally obtain the integral equation formulation of the Schrödinger-Newton- $\Lambda$ system as

$$
S(r)=S(0)-\int_{0}^{r} x\left(1-\frac{x}{r}\right) S(x) V(x) d x
$$

and

$$
V(r)=V(0)+\frac{\lambda r^{2}}{6}-\int_{0}^{r} x\left(1-\frac{x}{r}\right) S^{2}(x) d x,
$$

respectively. By taking the derivative of Eq. (20) with respect to $r$ we obtain

$$
V^{\prime}(r)=\frac{\lambda r}{3}-\int_{0}^{r} \frac{x^{2} S^{2}(x)}{r^{2}} d x=\frac{\lambda r}{3}-\phi(r),
$$

where $\phi(r)=\int_{0}^{r} x^{2} S^{2}(x) d x / r^{2}$. According to a standard result in calculus, if $f$ : $I \rightarrow R$ is a continuous and positive function on $I$, then $\int_{I} f(x) d x>0$. Since obviously $x^{2} S^{2}(x) / r^{2}>0$, it follows that $\phi(r)>0, \forall r>0$. Hence for $\lambda=0$, $V^{\prime}(r)<0$, and, therefore, in the absence of dark energy $V$ must be a monotonically decreasing function of $r$. However, there is a drastic change in the behavior of $V(r)$ in the presence of the cosmological constant $\lambda$. If $\lambda$ satisfies the condition $\lambda<$ $3 \phi(r) / r, \forall r \geq 0$, then, similarly to the standard Schrödinger-Newton case, $V^{\prime}(r)<$ $0, \forall r>0, V(r)$ is a monotonically decreasing function of the radial coordinate, and, if $V$ diverges at infinity, then $\lim _{r \rightarrow \infty} V(r)=-\infty$. On the other hand, if $\lambda>3 \phi(r) / r, \forall r \geq 0, V^{\prime}(r)>0, \forall r>0$, and $V(r)$ is a monotonically increasing function of $r$. Consequently, if $V$ is singular at infinity, then $\lim _{r \rightarrow \infty} V(r)=+\infty$. Generally, the rescaled gravitational potential $V(r)$ satisfies in the presence of the cosmological constant the condition

$$
\frac{d}{d r}\left[V(r)-V(0)-\frac{\lambda r^{2}}{6}\right]<0,
$$

which generalizes the condition $V^{\prime}(r)<0$ valid for the Schrödinger-Newton system. 


\subsection{The Adomian Decomposition Method}

We illustrate now the Adomian Decomposition Method for the case of a nonlinear second order ordinary differential equation, written in Adomian's operator-theoretic notation as $\sqrt{23} \sqrt{26}$

$$
L u(x)+R u(x)+N u(x)=g(x),
$$

where $g(x)$ is the system input, $u(x)$ is the system output, $L$ is the highest order differential operator, given, in our case, by $L()=.\frac{d^{2}}{d x^{2}}(),$.$R is the linear operator,$ while $N$ is the nonlinear operator, assumed to be analytic. In order to solve the initial value problem associated to Eq. (23), we adopt for the inverse linear operator $L^{-1}$ the two-fold definite integral $L^{-1}()=.\int_{a}^{x} \int_{a}^{x}() d x d$.$x , where a$ is the initial point. By applying the inverse of the operator $L$ to both sides of Eq. (23) we obtain the formal solution of the nonlinear differential equation as 23 26

$$
u=U+L^{-1} g-L^{-1}(R u+N u),
$$

where the first term $U$ in the above relation contains the initial conditions, and identically satisfies the equation $L U=0$.

The basic idea of the Adomian Decomposition Method is to represent $u(x)$ by the Adomian Decomposition series, $u(x)=\sum_{n=0}^{\infty} u_{n}(x)$, while the nonlinear term $N u$ is represented in terms of the Adomian polynomials $A_{n}(x)$, given by the formal expression, $N u(x)=\sum_{n=0}^{\infty} A_{n}(x)$. For a nonlinearity of the form $N u=f(u)$ the $A_{n}$ 's are defined according to 23 26

$$
A_{n}=A_{n}\left(u_{0}, u_{1}, \ldots, u_{n}\right)=\left.\frac{1}{n !} \frac{d^{n}}{d \lambda^{n}} f\left(\sum_{k=0}^{n} \lambda^{k} u_{k}(x)\right)\right|_{\lambda=0} .
$$

Substituting the Adomian expansions into Eq. (23) we obtain the following recursion scheme for the solution components,

$$
\begin{gathered}
u_{0}=U+L^{-1} g, \\
u_{n+1}=-L^{-1}\left(R u_{n}+A_{n}\right), n \geq 0 .
\end{gathered}
$$

Hence the $n+1$-term approximation of the solution is $u_{n+1}=\sum_{k=0}^{n} u_{k}$. In order to obtain a better approximation of the solution we will use the Padé approximants 27 of the Adomian series, which transform the polynomial approximations into a rational function that allows us to obtain more information about $u(x)$. The Padé approximants will converge on the entire real axis if $u(x)$ is free of singularities. 27

\section{Series solution of the Schrödinger-Newton- $\Lambda$ system via the Adomian Decomposition Method}

In the present Section we will consider a semianalytical approach to the SchrödingerNewton- $\Lambda$ system, by using the Adomian Decomposition Method and the Padé approximants. 
In order to apply these mathematical methods we will consider the equivalent mathematical formulations of the Schrödinger-Newton- $\Lambda$ system as a system of integral equations. In the following we will look for a series solution of the system (19) and (20), by assuming that $S(r)=\sum_{n=0}^{\infty} S_{n}(r)$, and $V(r)=\sum_{n=0}^{\infty} V_{n}(r)$, respectively. As for the nonlinear terms $S(x) V(x)$ and $S^{2}(x)$, we will decompose them in terms of the Adomian polynomials according to

$$
S(x) V(x)=\sum_{n=0}^{\infty} A_{n}(x), S^{2}(x)=\sum_{n=0}^{\infty} B_{n}(x)
$$

Substituting the above decompositions into Eqs. (19) and (20) we obtain

$$
\begin{gathered}
\sum_{n=0}^{\infty} S_{n}(r)=S(0)-\sum_{n=0}^{\infty} \int_{0}^{r} x\left(1-\frac{x}{r}\right) A_{n}(x) d x, \\
\sum_{n=0}^{\infty} V_{n}(r)=V(0)+\frac{\lambda r^{2}}{6}-\sum_{n=0}^{\infty} \int_{0}^{r} x\left(1-\frac{x}{r}\right) B_{n}(x) d x .
\end{gathered}
$$

Hence we obtain the following recursive relations for the determination of the solution of the Schrödinger-Newton- $\Lambda$ system,

$$
\begin{gathered}
S_{0}=S(0), S_{n+1}=-\int_{0}^{r} x\left(1-\frac{x}{r}\right) A_{n}(x) d x, \\
V_{0}=V(0)+\frac{\lambda r^{2}}{6}, V_{n+1}=-\int_{0}^{r} x\left(1-\frac{x}{r}\right) B_{n}(x) d x .
\end{gathered}
$$

As for the Adomian polynomials, they are given by $A_{0}=S(0) V_{0}, A_{1}=S(0) V_{1}+$ $S_{1} V_{0}, A_{2}=S(0) V_{2}+S_{1} V_{1}+S_{2} V_{0}$ etc., and $B_{0}=S^{2}(0), B_{1}=2 S(0) S_{1}, B_{2}=$ $2 S(0) S_{2}+S_{1}^{2}, B_{3}=2 S(0) S_{3}+2 S_{1} S_{2}$, respectively. Hence we obtain the first five successive terms in the Adomian series expansion of the Schrödinger-Newton- $\Lambda$ model as

$$
\begin{gathered}
S_{1}(r)=-\frac{1}{120} r^{2} S(0)\left[\lambda r^{2}+20 V(0)\right], \\
V_{1}(r)=-\frac{1}{6} r^{2} S^{2}(0), \\
S_{2}(r)=\frac{r^{4} S(0)\left\{\lambda r^{2} V(0)+42\left[S^{2}(0)+V^{2}(0)\right]\right\}}{5040}, \\
V_{2}(r)=\frac{r^{4} S^{2}(0)\left[\lambda r^{2}+42 V(0)\right]}{2520}, \\
362880
\end{gathered}
$$




$$
\begin{aligned}
V_{3}(r)= & -\frac{r^{6} S^{2}(0)\left\{63 \lambda^{2} r^{4}+4400 \lambda r^{2} V(0)+13200\left[3 S^{2}(0)+8 V^{2}(0)\right]\right\}}{99792000}, \\
S_{4}(r)= & \frac{r^{8} S(0)}{15567552000}\left\{393 \lambda^{2} r^{4} S^{2}(0)+78 \lambda r^{2} V(0)\left[557 S^{2}(0)+5 V^{2}(0)\right]+\right. \\
& \left.14300\left[27 S^{4}(0)+98 S^{2}(0) V^{2}(0)+3 V^{4}(0)\right]\right\}, \\
V_{4}(r)= & \frac{r^{8} S^{2}(0)}{7783776000}\left\{165 \lambda^{2} r^{4} V(0)+78 \lambda r^{2}\left[171 S^{2}(0)+191 V^{2}(0)\right]+\right. \\
& \left.114400 V(0)\left[5 S^{2}(0)+3 V^{2}(0)\right]\right\} .
\end{aligned}
$$

The next terms of the Adomian series expansion can be easily calculated. The Padé approximants of order [3/4] of the Adomian series truncated to the first six terms are given by

$$
\begin{aligned}
S(r)\left[\frac{3}{4}\right]= & \frac{1}{P(r)}\left\{60 r^{2} S(0) V(0)\left[-39 \lambda+23 S^{2}(0)-31 V^{2}(0)\right]-\right. \\
& \left.2520 S(0)\left[-3 \lambda+3 S^{2}(0)-7 V^{2}(0)\right]\right\},
\end{aligned}
$$

and

$$
V(r)\left[\frac{3}{4}\right]=\frac{X(r)}{Y(r)},
$$

respectively, where

$$
\begin{gathered}
P(r)=r^{4}\left\{63 \lambda^{2}+63 S^{4}(0)-2 S^{2}(0)\left[63 \lambda+32 V(0)^{2}\right]+33 V^{4}(0)-96 \lambda V^{2}(0)\right\}+ \\
120 r^{2} V(0)\left[-9 \lambda+S^{2}(0)+9 V^{2}(0)\right]-2520\left[-3 \lambda+3 S^{2}(0)-7 V^{2}(0)\right],(43) \\
X(r)=30\left\{r^{2}\left[35 S^{4}(0)-39 S^{2}(0) V^{2}(0)+8 V^{4}(0)-3 \lambda V^{2}(0)\right]+\right. \\
\left.42 V(0)\left[3 V^{2}(0)-5 S^{2}(0)\right]\right\},
\end{gathered}
$$

and

$$
\begin{aligned}
Y(r)= & 15 r^{4} S^{4}(0)+30 V(0)\left\{2 V(0)\left[4 r^{2} V(0)+63\right]-3 \lambda r^{2}\right\}- \\
& S^{2}(0)\left\{r^{4}\left[15 \lambda+23 V^{2}(0)\right]+540 r^{2} V(0)+6300\right\},
\end{aligned}
$$

respectively. 


\section{The numerical analysis of the $\mathrm{S}-\mathrm{N}-\Lambda$ system}

In the present Section we also perform a numerical analysis of the SchrödingerNewton- $\Lambda$ system, and compare the numerical results with the semianalytical approximations obtained via the Adomian Decomposition Method.

\subsection{Full numerical results}

We will present first some full numerical results that indicate the effects of the cosmological constant on the behavior of the solutions of the $\mathrm{S}-\mathrm{N}-\Lambda$ system. As it is already known from the numerical study of the Schrödinger-Newton system, it admits solutions for which $(S, V) \rightarrow( \pm \infty, \pm \infty)$. For example, for the initial conditions $S(0)=1.10$ and $V(0)=1$, the system blows up at $r \approx 10$ so that $S(r) \rightarrow+\infty$, and $V(r) \rightarrow-\infty$. However, with the inclusion of the cosmological constant in the model, both the quantitative and qualitative behavior of the model changes drastically, as shown in Fig. 1.
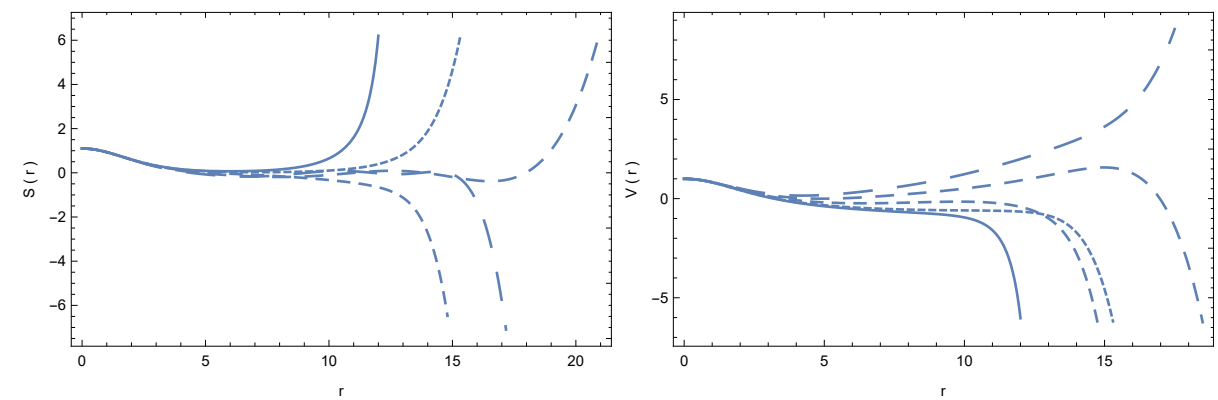

Fig. 1. The behavior of $S(r)$ (left panel) and $V(r)$ (right panel) as a function of $r$ (in arbitrary units) for $S(0)=1.10$ and $V(0)=1.0$, and for different values of the cosmological constant: $\lambda=0$ (solid curve), $\lambda=0.01$ (dotted curve), $\lambda=0.04$ (short dashed curve), $\lambda=0.08$ (dashed curve), and $\lambda=0.12$ (long dashed curve).

The first effect of the cosmological constant is the significant modification of the position of the blow-up point, which significantly increases with the increase of $\lambda$. More importantly, a set of different blow-up solutions with $S(r) \rightarrow-\infty$ and $V(r) \rightarrow+\infty$ do appear. The position of the first zeros of $S$ and $V$ are also displaced, and it increases with increasing $\lambda$.

The variations of $S$ and $V$ for $S(0)=0.50$ and $V(0)=1.0$ are represented in Fig. 2.

The presence of a cosmological constant has a significant impact on the behavior of the wave function, and on the gravitational potential energy. While the general oscillatory behavior of the wave function for large $r$ is not affected, the position of the zeros of $S$ depend on the value of the cosmological constant. The positions of the maximums and minimums of $S$ are also displaced as compared to the $\lambda=0$ case. The effect of the dark energy on the effective gravitational potential is very significant. 

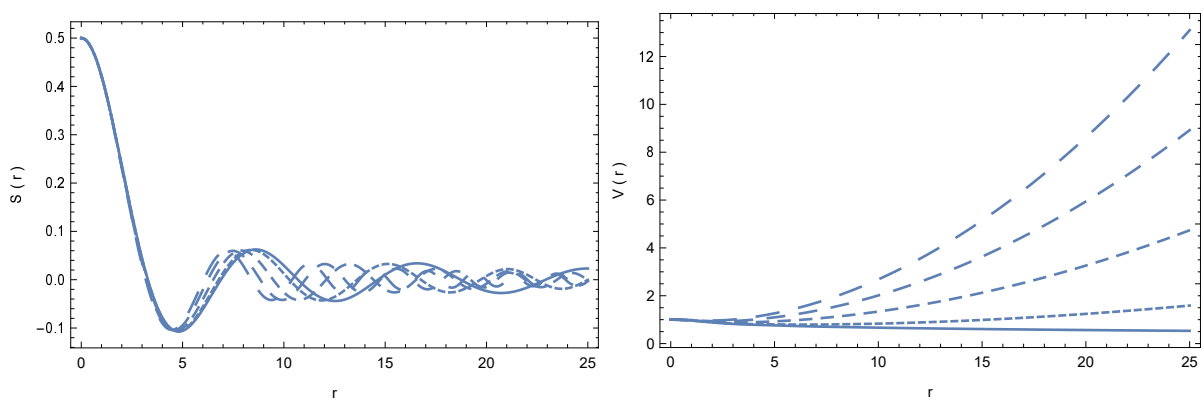

Fig. 2. The behavior of $S(r)$ (left panel) and $V(r)$ (right panel) as a function of $r$ (in arbitrary units) for $S(0)=0.5$ and $V(0)=1.0$, and for different values of the cosmological constant: $\lambda=0$ (solid curve), $\lambda=0.01$ (dotted curve), $\lambda=0.04$ (short dashed curve), $\lambda=0.08$ (dashed curve), and $\lambda=0.12$ (long dashed curve).

While for the simple S-N system $V$ is a monotonically decreasing function of $r$, tending to zero at infinity, for the adopted values of the cosmological constant, $V$ becomes a monotonically increasing function, diverging at infinity.

An interesting physical regime corresponds to the dark energy domination limit, corresponding to large values of $\lambda$. The comparison between the behavior of the wave function and gravitational potential of the N-S model with $\lambda=0$ and the dark energy dominated quantum system is presented for $S(0)=0.40, V(0)=1$, and for different large values of $\lambda$, in Fig. 3
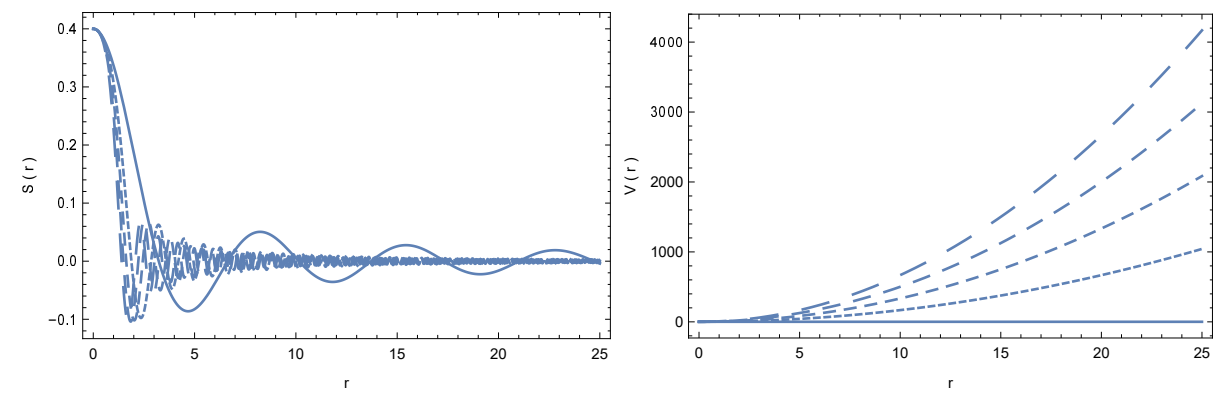

Fig. 3. The behavior of $S(r)$ (left panel) and $V(r)$ (right panel) as a function of $r$ (in arbitrary units) for $S(0)=0.4$ and $V(0)=1.0$, and for different values of the dark energy: $\lambda=0$ (solid curve), $\lambda=10$ (dotted curve), $\lambda=20$ (short dashed curve), $\lambda=30$ (dashed curve), and $\lambda=40$ (long dashed curve).

As one can see from the Figures, the presence of a large dark energy induces both qualitative and quantitative differences as compared to the behavior of the standard $\mathrm{S}-\mathrm{N}$ system. The oscillating behavior of the wave function is strongly modified, and in the presence of $\lambda$ the transition to zero takes place through a large number of oscillations. While for the S-N system $V$ is a slowly decreasing function of $r$, in the $\mathrm{S}-\mathrm{N}-\Lambda$ model $V(r)$ is a rapidly increasing function, taking numerical values three 
orders of magnitude higher than in the $\lambda=0$ case.

The matter density $\rho$ is defined quantum-mechanically according to $\rho=$ $m|\psi(\vec{r})|^{2}=\left(\hbar^{2} / 8 \pi G m^{2}\right) S^{2}(\vec{r})$. The variation of $S^{2}(r)$ as a function of the radial coordinate $r$ is represented, for different values of the cosmological constant $\lambda$, and for two particular set of initial conditions, in Fig. 4. We will first discuss the case $\lambda=0$, and $S(0)=0.4, V(0)=1.0$, respectively. The matter density has its maximum value at $r=0$, and it decreases rapidly with increasing $r$. However, after reaching a minimum value, the density increases again, attaining a second maximum with a much smaller amplitude, with this behavioral pattern repeating itself up to point where $\rho \approx 0$. We interpret these transitions from a decreasing to an increasing density as corresponding to the existence of a density bounce, and to the oscillations of the density of the quantum matter. This type of bouncing behavior is significantly affected by the presence of the large values of the cosmological constant. The matter density, having its maximum at $r=0$ independently of the absence or presence of the cosmological constant, reaches its first minimum value at much smaller values of $r$, as compared to the $\lambda=0$ case. Moreover, the successive maximums/minimums occur much closer to the origin, and a large number of density bounces do appear, corresponding to matter density oscillations, as compared to the few present in the $\lambda=0$ model. The bouncing behavior essentially depends not only on $\lambda$, but also on the initial conditions, as one can clearly see from the right panel of Fig. 4. For the initial values $S(r), S(0)=1.1, V(0)=1$, leading to the blow-up of the wave function, in the case $\lambda=0$ there is a clear density bounce, with the matter density decreasing to a minimum (almost zero) value, and then blowing up for larger values of $r$. This behavior, corresponding to a single bounce, and the appearance of a singularity in the matter density, is drastically modified by the presence of the cosmological constant that wipes out the singularity in the matter density. Hence, $\rho$ tends to zero through an oscillatory process, with the amplitude of the oscillations slowly decreasing with increasing $r$.
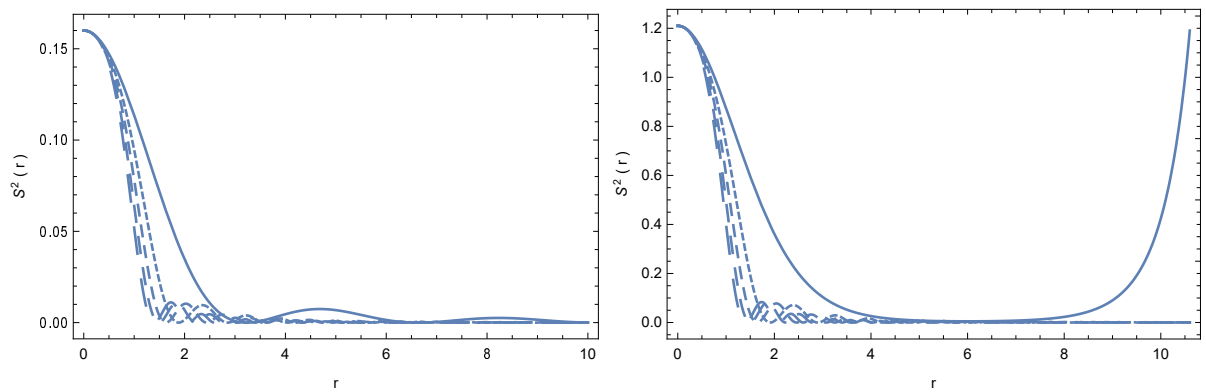

Fig. 4. Variation of the scaled matter energy density $S^{2}(r)$ as a function of $r$ (in arbitrary units) for $S(0)=0.4, V(0)=1.0$ (left panel) and for $S(0)=1.1, V(0)=1$ (right panel), and for different values of the dark energy: $\lambda=0$ (solid curve), $\lambda=10$ (dotted curve), $\lambda=20$ (short dashed curve), $\lambda=30$ (dashed curve), and $\lambda=40$ (long dashed curve). 


\subsection{Comparison with the semianalytical solutions}

We will consider now the comparison of the semianalytical solutions of the S-N$\Lambda$ system, obtained via the Adomian Decomposition Method-Padé approximants, and the full numerical solution. For the case of the S-N system, with $\lambda=0$, the comparison of the two solutions is represented, for $S(0)=1$ and $V(0)=1$, in Fig. 5
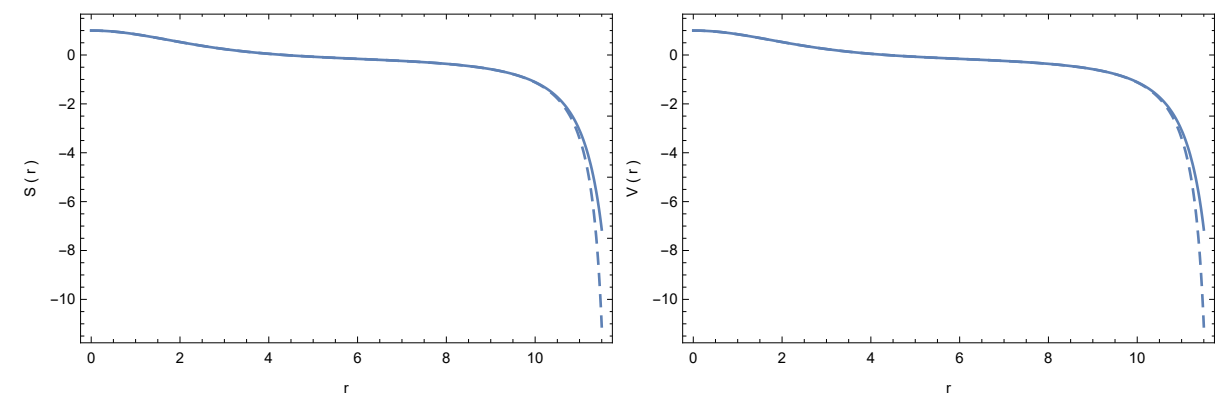

Fig. 5. Comparison of the semianalytical solution of the Newton-Schrödinger system (dashed curve), with $\lambda=0$, and the full numerical solution (solid curve), for $S(r)$ (left panel) and $V(r)$ (right panel) (in arbitrary units), for $S(0)=1$ and $V(0)=1$.

For the adopted initial conditions the semianalytical solution gives an excellent approximation of the numerical up to the appearance of the first singular point. For the case $\lambda=0.0001$, the comparison between the numerical and the semianalytical solution is presented in Fig. 6. for $S(0)=0.40$ and $V(0)=0.75$.
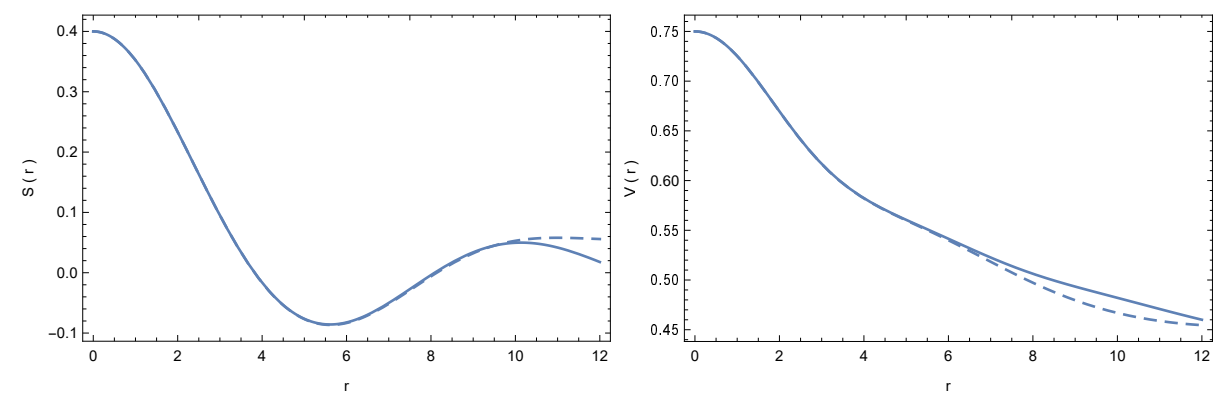

Fig. 6. Comparison of the semianalytical solution of the Newton-Schrödinger $-\Lambda$ system (dashed curve), and the full numerical solution (solid curve), for $S(r)$ (left panel) and $V(r)$ (right panel) (in arbitrary units), with $\lambda=0.0001$, and $S(0)=0.40$ and $V(0)=0.75$.

The comparison of the Adomian Decomposition semianalytical solution and the full numerical solution of the Schrödinger-Newton- $\Lambda$ system for $\lambda=1.4$ is represented in Fig. 7 .

For large values of $\lambda, S(0)$ and $V(0)$, we obtain a good approximation of the numerical solution in the range $0 \leq r \leq 1$. Even that generally the Adomian series 

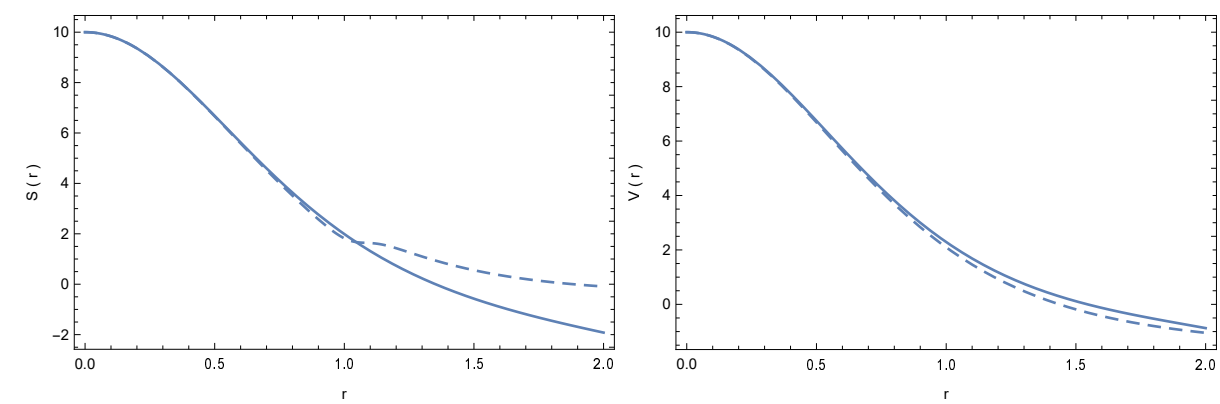

Fig. 7. Comparison of the semianalytical solution of the Newton-Schrödinger $-\Lambda$ system (dashed curve), and the full numerical solution (solid curve), for $S(r)$ (left panel) and $V(r)$ (right panel) (in arbitrary units), for $\lambda=1.4$, and $S(0)=10$ and $V(0)=10$, respectively.

does converge towards the exact/full numerical solution, for large values of $S(0)$, $V(0)$, and $\lambda$, the convergence is slow, and for the few terms considered in the series expansion, the semianalytical solution may describes well the full numerical solution only for small values of $r$, usually in the range $0 \leq r \leq 1$. For smaller values of $\lambda$ there is a good concordance between the numerical and the semianalytical approaches, and the two overlap even for large values of $r$. On the other hand it is important to point out that the Adomian Decomposition Method generally fails in the vicinity of the singular points of a differential equation, where the solution diverges.

Hence even in the presence of the cosmological constant, the Adomian Decomposition Methods, used together with the Padé approximants approach, provides a good approximation of the full numerical solution. The precision of the approximation essentially depends on the number of terms included in the Adomian decomposition, as well as of the order $m n$ of the Padé approximants. Moreover, the procedure can be easily implemented by using symbolic calculation software that allows to approximate the numerical solution with an arbitrary precision, thus helping in obtaining a deeper insight into its physical properties.

\section{Discussions and final remarks}

In the present Letter we have considered some basic properties of the simplest extension of the static Schrödinger-Newton system in spherical symmetry, which consists in the modification of the Poisson equation through the addition of the dark energy term, which we modeled as a simple cosmological constant. In the present model, the dynamical behavior of a quantum particle is determined by the nonlinear Schrödinger equation containing an effective potential including the standard Newtonian gravity and the dark energy contributions. After reformulating the $\mathrm{S}-\mathrm{N}-\Lambda$ system of two differential equations as an equivalent system of integral equations, we have applied the Adomian Decomposition Method to obtain a semianalytic power series solution. In order to avoid the oscillating/singular behaviors in the Adomian series we have approximated them as rational functions via the 
method of the Padé approximants. We have investigated the general S-N- $\Lambda$ system numerically, in order to point out the important role the dark energy may play in the quantum description of gravity, especially if interpreted as a vacuum fluctuation. The dark energy influences the number of zeros of the wave function, as well as the blow-up radius of the system. But in order to fully describe the effects of the dark energy a complete and systematic numerical and analytical investigation of the system is necessary. In particular, the novel and interesting aspects related to the energy spectrum must be carefully analyzed, and especially in the limit of a large cosmological constant value, the spectrum may give some insights into the quantum nature of gravity. For example, the problem of the existence of bound states at infinity takes a different form due to the presence of the nonzero cosmological constant at infinity. Moreover, the eigenvalue problem for large $r$, from which in principle one can determine exactly the eigenvalues, as well as the eigenfunctions asymptotically in the form of expansions of increasing accuracy becomes very different in the case of the $\mathrm{S}-\mathrm{N}-\Lambda$ system, as compared to the case of the $\mathrm{S}-\mathrm{N}$ system. The energy eigenvalues in the presence of the cosmological constant can be obtained from Eq. (12), by adopting, for example, for the first approximation of the ground state wave function the hydrogen atom wave function, $\psi(r)=\left(1 / \pi \sigma^{3}\right)^{1 / 2} e^{-r / \sigma}, \underline{42}$ which would allow to obtain perturbatively the energy eigenvalues, and the wave function in the presence of dark energy. But more precise predictions of the model also do depend on a full numerical study of the $\mathrm{S}-\mathrm{N}-\Lambda$ system in different physical contexts. The time-dependent $\mathrm{S}-\mathrm{N}-\Lambda$ system can also be investigated by using the Adomian Decomposition Method, and series solutions of the equation can be obtained easily 4

The present results on the existence/nonexistnce of a bounce in the matter density may have interesting cosmological implications. Bouncing solutions in which the Universe smoothly bounce from a collapsing to an expanding phase have attracted a lot of attention recently. A bouncing Universe does appear in Loop Quantum Cosmology, a quantum theory of gravity in which the macroscopic physical quantities (energy density, curvature, etc.), have finite upper bounds ${ }^{47}$ Hence, a contracting homogeneous and isotropic Universe will bounce back to an expanding one at finite values of the scale factor and energy density, thus preventing the appearance of a physical singularity. The blow-up of the solutions of the $\mathrm{S}-\mathrm{N}-\Lambda$ system for various initial conditions may, at first sight, suggest that Newtonian quantum gravity cannot consistently solve the cosmological problem, and a fine-tuning of the initial conditions is necessary. However, in order to give a full answer to this question the investigation of the cosmological behavior of the time-dependent $\mathrm{S}-\mathrm{N}-\Lambda$ is necessary, in which the effects of classical general relativity are also included. Such a study may lead to a better understanding of the quantum gravity aspects in the early Universe, and provide observational signatures of quantum gravity, which may have some observational imprints on the primordial power spectrum of the Cosmic Background Microwave Radiation. 
Moreover, the S-N- $\Lambda$ model opens the possibility for the understanding of the quantum to classical transition in the presence of dark energy, which provides a new effect not directly related to the increase of the mass of the particle. And, equally importantly, this quantum model combining classical gravity and quantum mechanics allows the investigation of quantum situations in which not only the gravitational field but also quantum fluctuations (interpreted as a dark energy) play a dominant role.

\section{Acknowledgments}

We would like to thank the two anonymous referees for comments and suggestions that helped us to improve our manuscript.

\section{References}

1. L. Modesto and L. Rachwal, International Journal of Modern Physics D 26, 1730020564 (2017).

2. G. Gubitosi, C. Ripken, and F. Saueressig, Foundations of Physics 49, 972 (2019).

3. R. Loll, Classical and Quantum Gravity 37, 013002 (2020).

4. S. Steinhaus, Frontiers in Physics 8, 295 (2020). .

5. L. Diósi and B. Lukács, Annalen der Physik 499, 488 (1987).

6. R. Penrose, Phil. Trans. R. Soc. Lond. A 356, 1927 (1998).

7. D. M Lan, Modern Physics Letters A 14, 2667 (1999).

8. L. Diósi, Foundations of Physics 44, 483 (2014).

9. A. Grossardt and B. C. Hiesmayr, Phys. Rev. D 91, 064056 (2015).

10. S. Bera, R. Mohan, and T. P. Singh, Phys. Rev. D 92, 025054 (2015).

11. S. Bera, S. Donadi, K. Lochan, and T. P. Singh, Foundations of Physics 45, 1537 (2015).

12. G. M. Prosperi, Foundations of Physics 46, 1426 (2016).

13. S. Bera, P. Giri, and T. P. Singh, Foundations of Physics 47, 897 (2017).

14. A. Tilloy and L. Diósi, Phys. Rev. D 96, 104045 (2017).

15. R. Howl, R. Penrose, and I. Fuentes, New Journal of Physics 21, 043047 (2019).

16. L. Diósi, J. Phys. Conf. Ser. 1275, 011001 (2019).

17. B. K. Lotte and S. Mishra, Modern Physics Letters A 35, 2050081 (2020).

18. X. Liu, T. Harko, and S.-D. Liang, The European Physical Journal C 76, 420 (2016).

19. T. D. Ferreira, N. A. Silva, O. Bertolami, C. Gomes, and A. Guerreiro, Phys. Rev. E 101, 023301 (2020).

20. Kelvin, K. Onggadinata, M. J. Lake, and T. Paterek, Phys. Rev. D 101, 063028 (2020).

21. L. Amendola and S. Tsujikawa, Dark Energy, Theory and Observations, Cambridge University Press, Cambridge, England, 2010

22. T. Harko and F. S. N. Lobo, Int. J. Mod. Phys. D 29, 2030008 (2020).

23. G. Adomian, J. Comput. Appl. Math. 11, 2 (1984).

24. G. Adomian, J. Math. Anal. Appl. 135, 501 (1988).

25. G. Adomian, Solving Frontier Problems of Physics: the Decomposition Method, Kluwer, Dordrecht, 1994

26. G. Adomian and R. Rach, Mathematical and Computer Modelling 24, 39 (1996).

27. A.-M. Warwaz, Appl. Math. Comput. 105, 11 (1999).

28. A.-M. Warwaz, Appl. Math. Comput. 102, 77 (1999). 
29. A.-M. Wazwaz, R. Rach and J.-S. Duan, Math. Meth. Appl. Sci. 37, 10 (2014).

30. H. Ghasemi, M. Ghovatmand, S. Zarrinkamar, and H. Hassanabadi, The European Physical Journal Plus 129, 32 (2014).

31. R. Rach, A.-M. Wazwaz and J.-S. Duan, J. Appl. Math. Comput. 47, 365 (2015).

32. J.-S. Duan, R. Rach, and A.-M. Wazwaz, International Journal of Computer Mathematics 94, 197 (2017).

33. M. K. Mak, C. S. Leung, and T. Harko, Advances in High Energy Physics 2018, 7093592 (2018).

34. M. K. Mak, C. S. Leung, and T. Harko, Surveys in Mathematics and its Applications 13, 183 (2018)

35. T. Harko, M. K. Mak, and C. S. Leung, Romanian Reports in Physics 72, 116 (2020).

36. T. Harko and M. K. Mak, accepted for publication in Romanian Reports in Physics, arXiv:2006.07170 [q-bio.PE] (2020).

37. T. Harko and M. K. Mak, arXiv:2009.00434 [q-bio.PE] (2020).

38. T. Harko, M. K. Mak, and M. J. Lake, arXiv:2011.11072 [gr-qc] (2020).

39. R. Penrose, Phil. Trans. R. Soc. 356, 1 (1998).

40. I. M. Moroz, R. Penrose, and K. P. Tod, Class. Quantum Grav. 15, 2733 (1998).

41. K. P. Tod and I. M. Moroz, Nonlinearity 12, 201 (1999).

42. D. Kumar and V. Soni, Physics Letters A 271, 157 (2000).

43. K. P. Tod, Physics Letters A 280, 173 (2001).

44. R. Harrison, I. Moroz, and K. P. Tod, Nonlinearity 16, 101 (2003).

45. J. Wei and M. Winter, Journal of Mathematical Physics 50, 012905-012905-22 (2009).

46. C. Duval and S. Lazzarini, Classical and Quantum Gravity 32, 175006 (2015).

47. Y. Ye, T. Harko, and S.-D. Liang, The European Physical Journal C 78, 587 (2018).

48. T. Harko, M. K. Mak, and M. J. Lake, arXiv:2011.11072 [gr-qc] (2020). 